\title{
Review Article \\ Efficacy of Suxiao Jiuxin Pill on Coronary Heart Disease: A Meta-Analysis of Randomized Controlled Trials
}

\author{
Li Ren, Jie Wang (D), Ling Feng, Shuli Wang, and Jun Li \\ Department of Cardiovascular, Guanganmen Hospital, China Academy of Chinese Medical Sciences, Beijing 100053, China \\ Correspondence should be addressed to Jie Wang; wangjie0103@126.com
}

Received 15 July 2017; Accepted 29 January 2018; Published 27 March 2018

Academic Editor: I-Min Liu

Copyright (C) 2018 Li Ren et al. This is an open access article distributed under the Creative Commons Attribution License, which permits unrestricted use, distribution, and reproduction in any medium, provided the original work is properly cited.

\begin{abstract}
Suxiao jiuxin pill is considered an effective ancillary drug in patients with coronary heart disease. Although numerous small, single-center clinical trials have been conducted, the benefits and harms of suxiao jiuxin pill remain controversial. We performed a meta-analysis to clarify the efficacy of suxiao jiuxin pill on patients with coronary heart disease. Randomized controlled trials were identified by using the Cochrane Library, PubMed, Web of Science, Embase, Wanfang, Weipu, and China Knowledge Resource Integrated databases (until June 2016). Pooled relative risks (RR), weighted mean differences (WMD), and 95\% confidence intervals (95\% CIs) were estimated using random-effects models. Forty-one trials involving 6276 patients were included in our analysis. Administration of suxiao jiuxin pill significantly improved electrocardiogram (ECG) results when compared with other therapies (RR 1.32, 95\% CI 1.26 to 1.38, and $P<0.001$ ). Subgroup analyses revealed that suxiao jiuxin pills improve ECG results more than salvia tablets (RR $1.54,95 \%$ CI 1.41 to 1.67 , and $P<0.001$ ), isosorbide dinitrate (RR 1.14, 95\% CI 1.21 to 1.44 , and $P=0.001$ ), nitroglycerin (RR $1.35,95 \%$ CI 1.16 to 1.56 , and $P<0.001$ ), and other drugs (RR 1.32 , $95 \%$ CI 1.21 to 1.44 , and $P<0.001$ ). Available evidence additionally suggests that suxiao jiuxin pills could significantly reduce total cholesterol (WMD $-0.62 \mathrm{mmol} / \mathrm{L}, 95 \% \mathrm{CI}-1.06$ to $-0.18 \mathrm{mmol} / \mathrm{L}$, and $P=0.005)$ and low-density lipoprotein (LDL) levels (WMD $-1.12 \mathrm{mmol} / \mathrm{L}$, 95\% CI -1.42 to $-0.82 \mathrm{mmol} / \mathrm{L}$, and $P<0.001$ ) and increase high-density lipoprotein (HDL) levels (WMD $0.32 \mathrm{mmol} / \mathrm{L}, 95 \%$ CI 0.07 to $0.58 \mathrm{mmol} / \mathrm{L}$, and $P=0.014$ ). However, no significant differences were observed in total triglyceride levels, plasma viscosity, hematocrit, and fibrinogen. No incidences of adverse reactions were observed after administration of suxiao jiuxin pill. Improvements in ECG results and lipid profiles were also observed after suxiao jiuxin administration compared to other therapies. It also decreased low-cut and high-cut whole blood viscosity without significant adverse reactions.
\end{abstract}

\section{Introduction}

Coronary heart disease (CHD) has become the leading cause of death in both men and women worldwide [1]. Most CHDrelated deaths occur in individuals older than 65 years of age. The spectrum of CHD includes subclinical CHD, chronic stable angina pectoris, unstable angina, and acute myocardial infarction. A large number of elderly patients have asymptomatic heart disease; therefore, the prevalence of $\mathrm{CHD}$ may be underestimated [2]. Several large prospective clinical studies [3-7] have demonstrated that CHD is significantly associated with atrial fibrillation, congestive heart failure, stroke, and other serious diseases. Hence, it is important to develop effective therapies to mitigate the progression of this disease.
Suxiao jiuxin pills are one of the most commonly used Chinese medicines for cardiocerebral vascular conditions. They were first developed by Chinese medicine specialist Chenggui Zhang in the 1980s and manufactured by the Sixth Chinese Drugs Factory of Tianjin Zhongxin Pharmaceutical Co., Ltd. [8, 9]. Small doses of suxiao jiuxin pill have been shown to rapidly relieve angina pectoris and improve its symptoms without any obvious side effects. Several reports have suggested that suxiao jiuxin pill helps lower the patients' lipid profile and improve myocardial function [10]. The main components of suxiao jiuxin pills are borneol and Ligusticum chuanxiong Hort [9, 11-15]. These ingredients can effectively induce relaxation and inhibit artery contraction $[9,11]$. In addition, several smaller clinical studies have been conducted to study the efficacy of suxiao jiuxin pill on CHD 
patients; however, these results have been inconsistent [1619]. Cao and Zhang suggested that suxiao jiuxin pill was associated with symptom remission, reduced incidence of angina, and shorter duration of angina. Further, electrocardiogram results were significantly improved by nitroglycerin use compared with salvia [16]. Qiao et al. demonstrated that suxiao jiuxin pills plus trimetazidine therapy significantly reduced the effective rate of angina, but other relevant indices were not evaluated [17-19]. Clarifying the beneficial and harmful effects of suxiao jiuxin pill is particularly important for $\mathrm{CHD}$ patients, as they have not been distinctly determined with respect to ECG results, lipid profiles, hemorheology, and adverse reactions. Therefore, we performed a large-scale meta-analysis of the available randomized controlled trials to determine the benefits of suxiao jiuxin pill for CHD patients.

\section{Materials and Methods}

Ethical approval and written consent were not necessary for the meta-analysis, as the data was collected from published literature.

Our meta-analysis was conducted according to the Preferred Reporting Items for Systematic Reviews and MetaAnalyses guidelines [20]. We searched the Cochrane Library, PubMed, Web of Science, Embase, Wanfang, Weipu, and China Knowledge Resource Integrated databases to identify relevant studies published in English or Chinese prior to June 2016. Our search terms included "coronary heart disease," "suxiao jiuxin pill," and "coronary artery disease." We also searched for meta-analysis publications and bibliographies referenced in the selected publications. Gray literature was identified through related agencies and clinical trial registers. Clinical trials that compared the efficacy of suxiao jiuxin pill on coronary heart disease with those of placebo or standard therapy were included in this meta-analysis. Criteria for inclusion were as follows: (1) a randomized controlled study design, (2) the possibility of extracting accurate clinical data, (3) classifying coronary heart disease based on the updated guidelines of the American Heart Association and American College of Cardiology Foundation [21, 22], and (4) reporting ECG results, lipid profiles, and/or hemorheology changes as outcomes. Two reviewers (X. L. H. and J. Z. J.) independently reviewed the studies to determine whether they satisfied the eligibility criteria. Discrepancies between reviewers' opinions were resolved by consensus, and a third reviewer was consulted when necessary.

2.1. Data Extraction. Two independent reviewers using the same checklist evaluated the data from the included studies. Disagreements between the reviewers were resolved by discussion until consensus was reached. The following sets of data were extracted for each selected study when available: demographics and sample characteristics, definition of coronary heart disease, and usage of suxiao jiuxin pill. The primary outcome of the selected studies was the improvement in ECG results, including resting ECG returning to normal or negative submaximal exercise test. The secondary outcomes included changes in lipid profile (total cholesterol, total triglyceride, low-density lipoprotein, and high-density lipoprotein) and hemorheology (high-cut whole blood viscosity, low-cut whole blood viscosity, plasma viscosity, hematocrit, and fibrinogen) as well as any adverse reactions.

2.2. Quality Assessment. Two reviewers independently assessed the methodological quality of the studies using the Jadad scoring system [23]. Five aspects for each study were thoroughly evaluated: the statement of randomization, the method used for generating randomized sequences for treatment assignments, the use of double-blind design, the description of the double blinding method, and data on withdrawals and dropouts. Studies with a score less than 3 were considered as low quality studies with high bias risk. Studies that received a score of 3 or greater were considered as high-quality studies. Disagreements between the reviewers were resolved by consensus and consultation with a third reviewer when necessary.

2.3. Data Analysis. Continuous variables, such as changes in lipid profiles and hemorheology, were expressed as mean \pm standard deviation. Categorical data, such as ECG result improvement and adverse reaction incidence, were presented as frequencies and percentages. We computed the pooled relative risk (RR), weighted mean difference (WMD), and 95\% confidence interval (CI), as well as the heterogeneity of the included studies by using random-effect (DerSimonian and Laird) models. Metaregression analysis was conducted based on sample size and mean age to explore the impact of sample size on the source of heterogeneity [24]. We also performed subgroup analyses to compare the efficacy of different drugs with those of suxiao jiuxin pill on ECG result improvement. Heterogeneity was quantified using the $I^{2}$ statistic. We considered $I^{2}$ values greater than $50 \%$ to indicate significant heterogeneity between the studies. Statistical heterogeneity between studies was also formally tested with the Cochran test $(P<0.10)[25,26]$. Publication bias was evaluated using the funnel plot and Egger's and Begg tests, with $P$ values less than 0.05 considered significant publication bias. Two-tailed $P$ values less than 0.05 were considered statistically significant. All statistical analyses were performed with STATA 12.0 (Stata Corporation, College Station, TX, USA).

\section{Results}

3.1. Search Results. The search strategy revealed 1515 potentially eligible publications. After duplicate removal, 1253 studies remained. Abstracts were evaluated based on the inclusion and exclusion criteria. 85 studies warranting further review were identified. Among these, 44 studies were excluded for the reasons listed in Figure 1. The remaining 41 studies were included in our meta-analysis. Journal articles and full manuscripts were obtained for all 41 studies.

3.2. Study Characteristics. The characteristics of the trials included in our meta-analysis are presented in Table 1 . All of the included studies were conducted in China. The following studies were included as the control group: 2 studies involving 


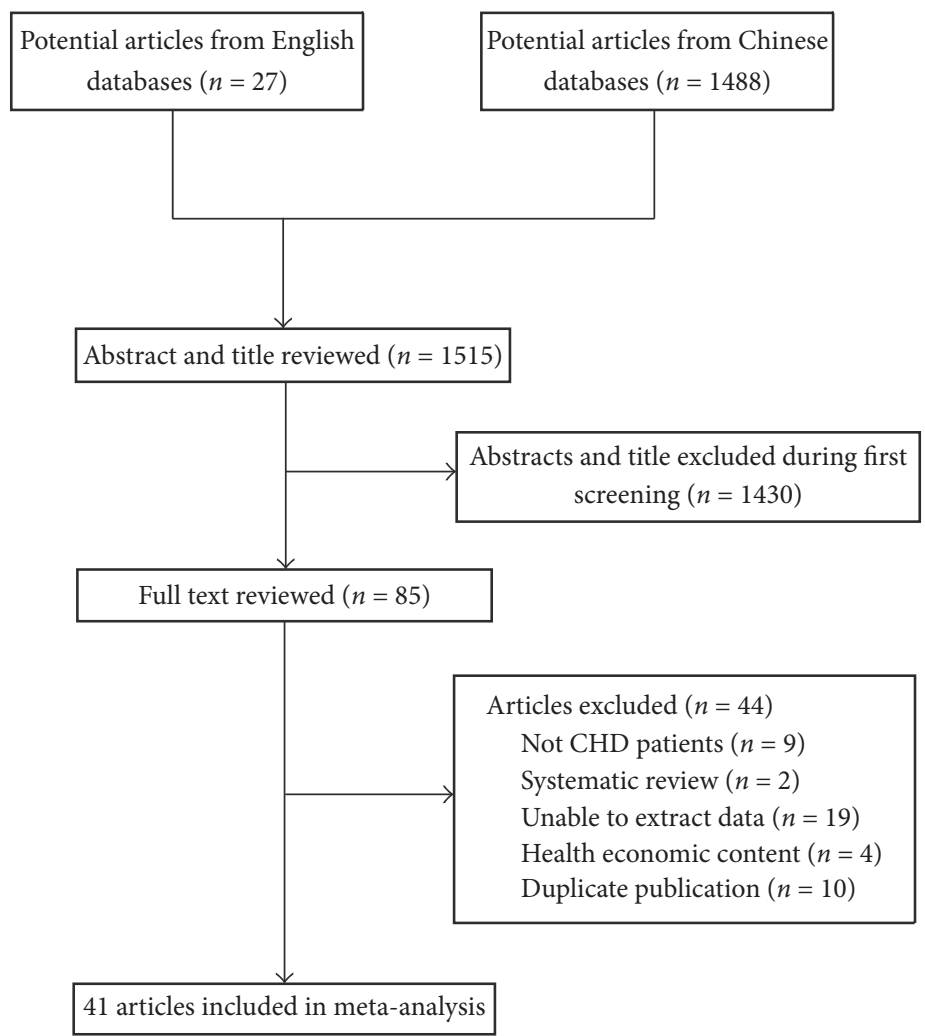

Figure 1: Process used to select relevant studies for inclusion in the meta-analysis.

standard treatment [27, 28], 7 studies involving nitroglycerin (using various formulations) [29-35], 12 studies involving isosorbide dinitrate [36-47], 11 studies involving salvia tablets $[16,17,48-56]$, and 9 studies involving Chinese herbal pills other than suxiao jiuxin $[18,32,57-64]$.

The 41 studies consisted of 6276 patients with coronary heart disease. The mean age in the treatment group was $57.57 \pm 8.15$ years and $54.10 \%$ of the patients were male. The mean age in the control group was $57.80 \pm 8.72$ years and $45.96 \%$ of the patients were male. The baseline characteristics were balanced between the treatment and control groups. The majority of the included studies received low Jadad scores due to the lack of a double-blind design (Table 2).

3.3. ECG Result Improvement. Figure 2 presents the results of the meta-analysis of ECG improvement following administration of suxiao jiuxin pills. ECG results were reported in 35 studies. Pooled analysis indicated significant benefits of suxiao jiuxin pill on ECG outcomes (RR 1.32; 95\% CI 1.26 to $1.38, P<0.001)$. However, there was significant heterogeneity between studies with respect to ECG results $\left(I^{2}=62.3 \%\right)$. Findings from the meta-regression analyses suggested that sample size and mean age of the patients were not significant factors contributing to the association between suxiao jiuxin pills and ECG outcomes (Table 3). Considering that the control group may be the source of the heterogeneity, we performed subgroup analyses to compare the effects of suxiao jiuxin pill with those of control treatment (Figure 3). The subgroup analyses indicated that suxiao jiuxin pill improved
ECG results more than salvia tablets (RR 1.54; 95\% CI 1.41 to $1.67, P<0.001$ ), isosorbide dinitrate (RR 1.14; $95 \% \mathrm{CI}$ 1.05 to $1.22, P=0.001$ ), nitroglycerin (RR $1.35,95 \% \mathrm{CI}$ 1.16 to $1.56, P<0.001$ ), and other drugs (RR $1.32,95 \% \mathrm{CI}$ 1.21 to $1.44, P<0.001)$. The $I^{2}$ decreased to $39.1 \%$ in the nitroglycerin subgroup. However, moderate heterogeneity was still observed among the other three subgroups $\left(I^{2}=\right.$ $50.0 \%, 44.9 \%$, and $43.6 \%$, resp.). Sensitivity analysis was conducted by excluding individual studies one after another but did not reveal a substantial change in the overall trend of heterogeneity between studies. We also constructed a funnel plot to assess the degree of publication bias. The funnel plot was symmetrically distributed around the pooled effect size, which indicated the absence of significant publication bias in the included studies (Figure 4). In addition, examining the funnel plot asymmetry via Egger test $(P=0.067)$ and Begg test $(P=0.050)$ did not demonstrate publication bias.

3.4. Lipid Profile. Only four of the studies reported the efficacy of suxiao jiuxin pill on patients' lipid profiles, and they all reported the total cholesterol in the patients' serum [33, 55, 57, 63]. Significant lower total cholesterol levels were reported in the suxiao jiuxin pill group compared with the control group (WMD $-0.62 \mathrm{mmol} / \mathrm{L}, 95 \% \mathrm{CI}-1.06$ to $-0.18 \mathrm{mmol} / \mathrm{L}$, and $P=0.005)$. There was significant heterogeneity among the 4 studies with respect to total cholesterol levels $\left(I^{2}=77.1 \%\right.$ ) (Supplemental Figure S1). In addition, the four studies reported total triglyceride levels in the patients' serum $[33,55,57,63]$. There was no significant 


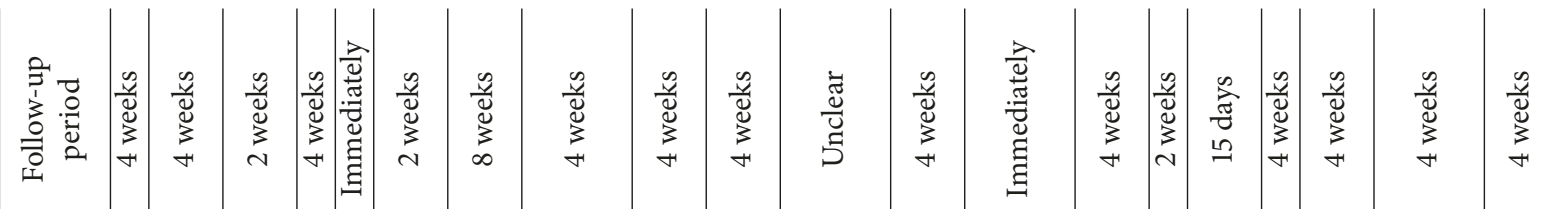

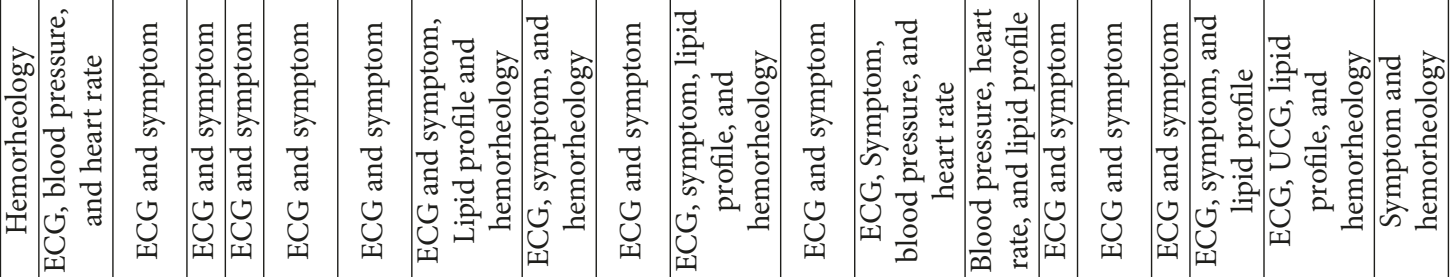

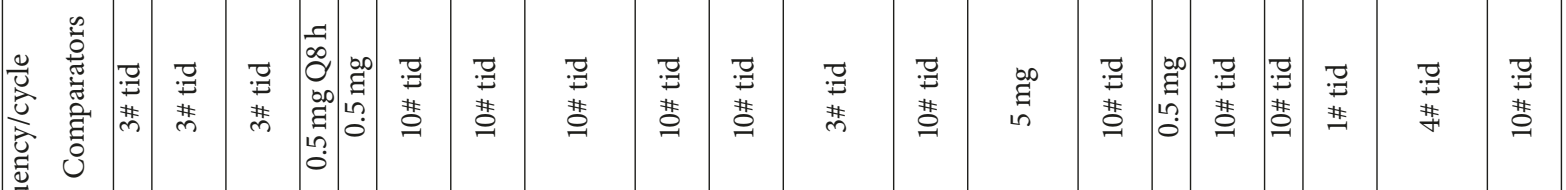

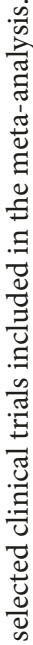
莺素

言 竎

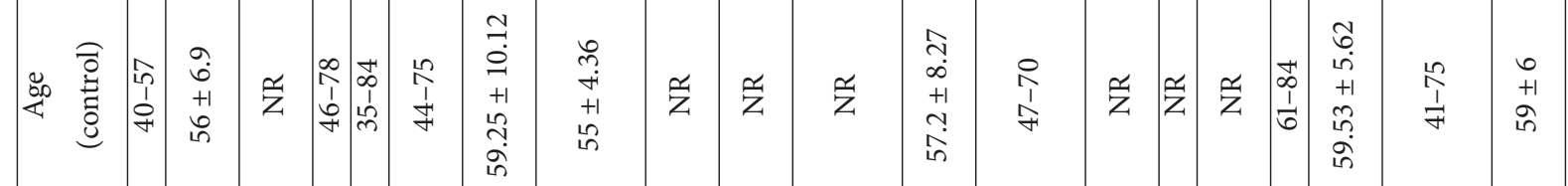

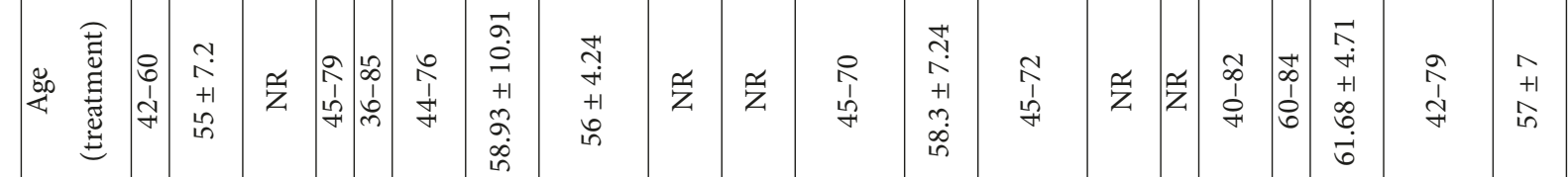
害总 䔍芯 芴

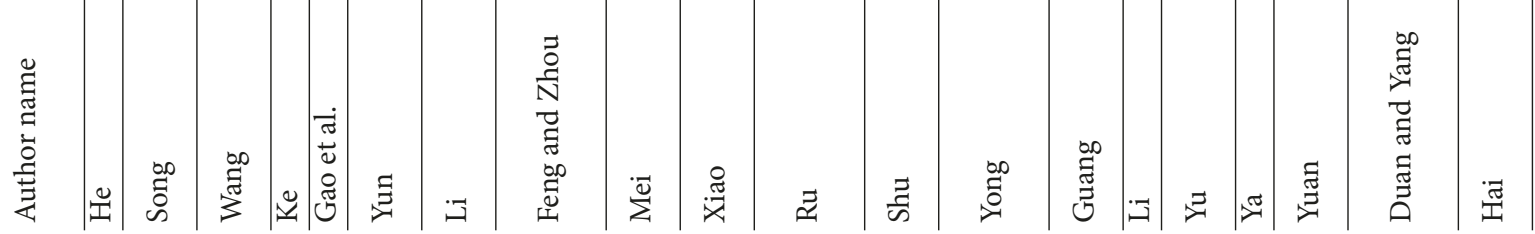




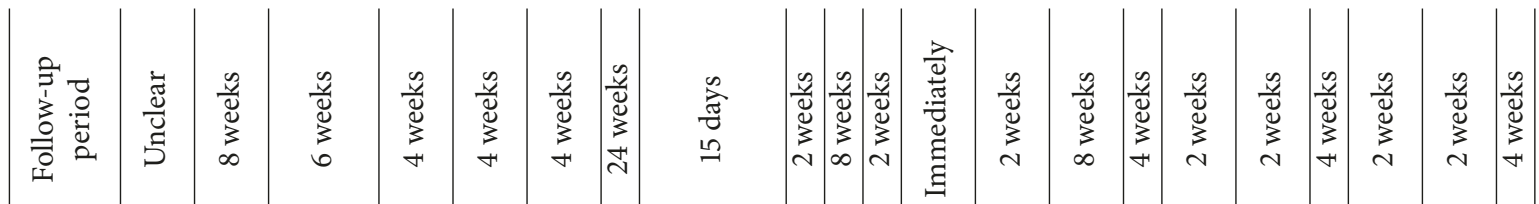

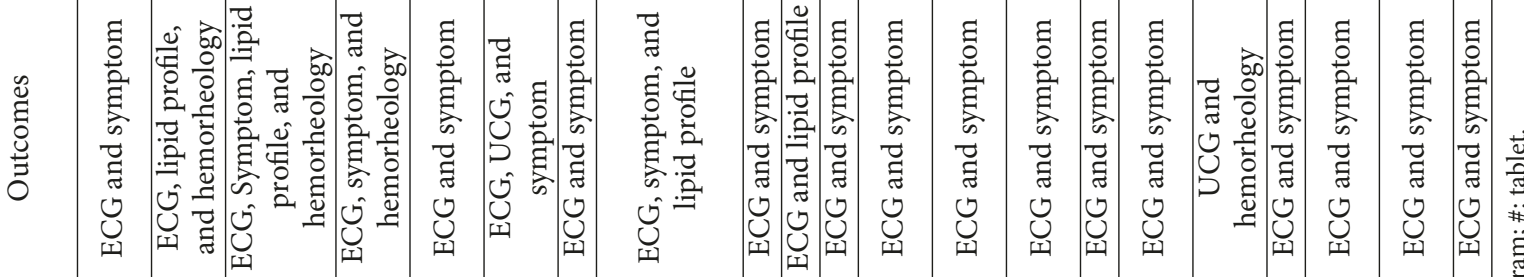

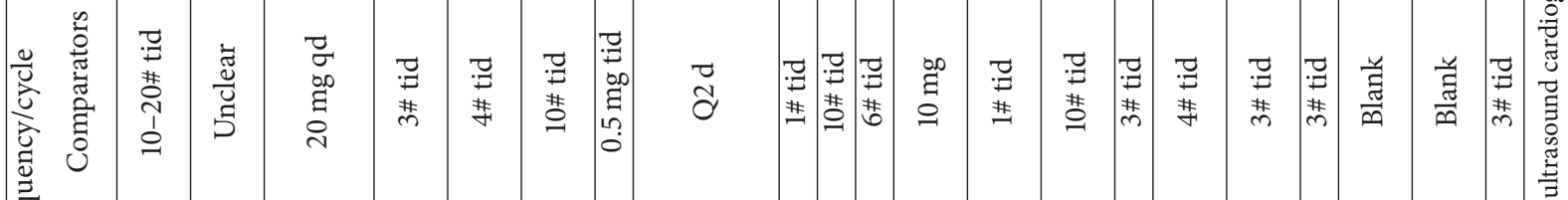

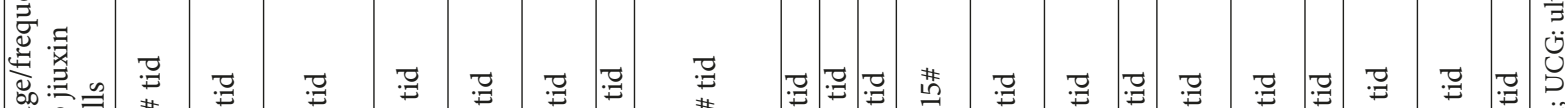

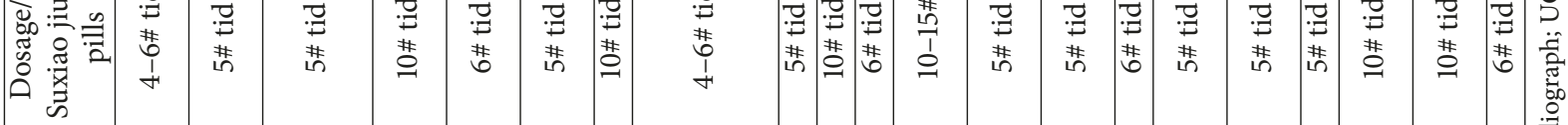
总

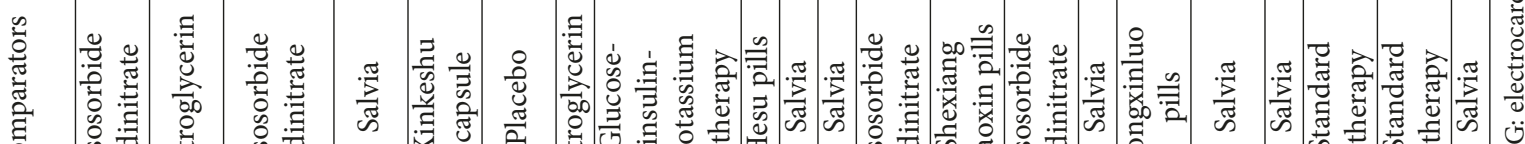

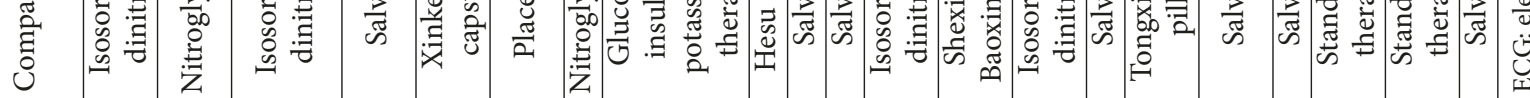
至

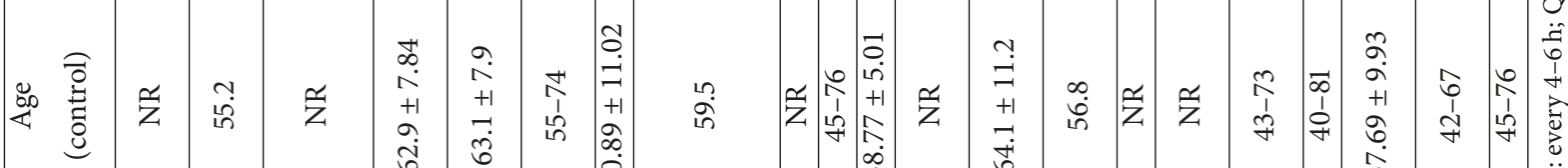

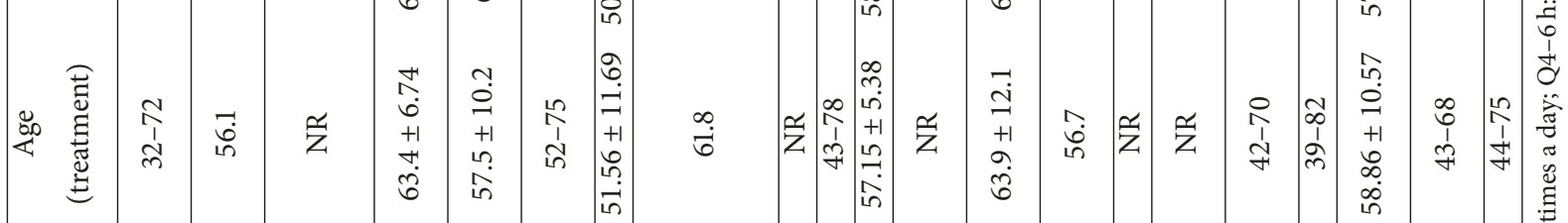

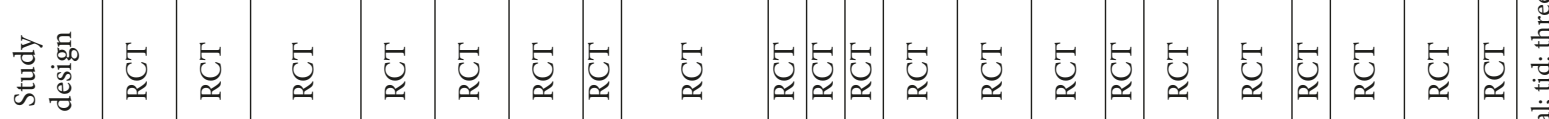
言: 荧

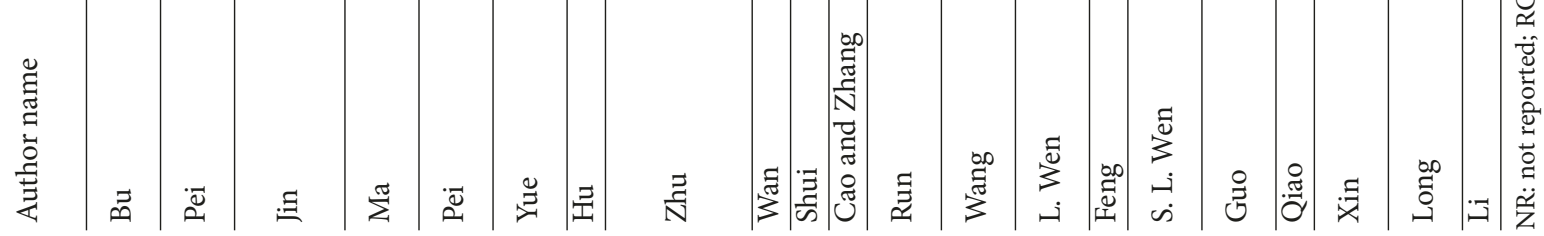


TABLE 2: Jadad quality scores of selected studies.

\begin{tabular}{|c|c|c|c|c|c|}
\hline Author name & Randomization & $\begin{array}{l}\text { Generating randomized } \\
\text { sequences }\end{array}$ & Blinding & $\begin{array}{l}\text { Withdrawals and } \\
\text { dropouts }\end{array}$ & Overall \\
\hline Guan-hua He & 1 & 0 & 0 & 0 & 1 \\
\hline Zhi-jin Song & 1 & 0 & 0 & 0 & 1 \\
\hline Dong-ping Wang & 1 & 0 & 1 & 0 & 2 \\
\hline Ke-fu Ji & 1 & 0 & 0 & 0 & 1 \\
\hline Yu-chu Gao & 1 & 0 & 0 & 0 & 1 \\
\hline Yun-yuan Guo & 1 & 0 & 0 & 0 & 1 \\
\hline Li An & 1 & 0 & 0 & 0 & 1 \\
\hline Ling Feng & 1 & 0 & 0 & 0 & 1 \\
\hline Mei $\mathrm{Hu}$ & 1 & 0 & 0 & 1 & 2 \\
\hline Xiao-chun Liu & 1 & 0 & 0 & 0 & 1 \\
\hline Ru-bao Jia & 1 & 0 & 0 & 0 & 1 \\
\hline Shu-dong Yang & 1 & 0 & 0 & 0 & 1 \\
\hline Yong-jin Hou & 1 & 0 & 0 & 0 & 1 \\
\hline Guang-yu Tang & 1 & 0 & 0 & 0 & 1 \\
\hline Li-jun Zhou & 1 & 0 & 0 & 0 & 1 \\
\hline Yu-ping Li & 1 & 0 & 0 & 0 & 1 \\
\hline Ya-xiong Zhan & 1 & 0 & 1 & 0 & 2 \\
\hline Jing-xian Yuan & 1 & 0 & 0 & 0 & 1 \\
\hline Ke-jie Duan & 1 & 0 & 0 & 0 & 1 \\
\hline Hai Shi & 1 & 0 & 0 & 0 & 1 \\
\hline Bu-ce Sun & 1 & 0 & 0 & 0 & 1 \\
\hline Pei-ying Wu & 1 & 0 & 0 & 0 & 1 \\
\hline Jin Gao & 1 & 0 & 0 & 0 & 1 \\
\hline Xian-zhen Ma & 1 & 0 & 0 & 0 & 1 \\
\hline Pei-fen Chang & 1 & 0 & 0 & 0 & 1 \\
\hline Yue-sheng Zhao & 1 & 0 & 0 & 0 & 1 \\
\hline Gang $\mathrm{Hu}$ & 1 & 0 & 0 & 0 & 1 \\
\hline Dong-you Zhu & 1 & 0 & 0 & 0 & 1 \\
\hline Wei Wan & 1 & 0 & 0 & 0 & 1 \\
\hline Shui-xiang Wan & 1 & 0 & 0 & 0 & 1 \\
\hline Sheng-hai Cao & 1 & 0 & 0 & 0 & 1 \\
\hline Run-lian Tang & 1 & 0 & 0 & 1 & 2 \\
\hline Fei Wang & 1 & 0 & 0 & 0 & 1 \\
\hline Wen Luo & 1 & 0 & 0 & 0 & 1 \\
\hline Feng-hua Song & 1 & 0 & 0 & 0 & 1 \\
\hline Wen-sheng Li & 1 & 0 & 1 & 0 & 2 \\
\hline Wei-qin Guo & 1 & 0 & 0 & 0 & 1 \\
\hline Qiao-kun Xu & 1 & 0 & 0 & 0 & 1 \\
\hline Xin $\mathrm{He}$ & 1 & 0 & 0 & 0 & 1 \\
\hline Long-jiang Qian & 1 & 0 & 0 & 0 & 1 \\
\hline Li Xiao-jin & 1 & 0 & 0 & 1 & 2 \\
\hline
\end{tabular}

difference in WMD between the treatment and control groups (WMD $-0.59 \mathrm{mmol} / \mathrm{L}, 95 \% \mathrm{CI}-1.72$ to $0.54 \mathrm{mmol} / \mathrm{L}$, and $P=0.303)$. We detected significant heterogeneity between studies among the 4 studies with respect to total triglyceride levels $\left(I^{2}=98.2 \%\right)$ (Supplemental Figure S2). Three of the studies reported LDL levels [33, 57, 63]. There were significantly lower LDL levels in the treatment group compared with the control group (WMD $-1.12 \mathrm{mmol} / \mathrm{L}, 95 \%$ CI -1.42 to $-0.82 \mathrm{mmol} / \mathrm{L}$, and $P<0.001)$ and lower heterogeneity between the 3 trials $\left(I^{2}=56.5 \%\right)$ (Supplemental Figure S3). Furthermore, the four studies reported HDL levels $[30,52,54,60]$. There were significantly higher HDL levels 


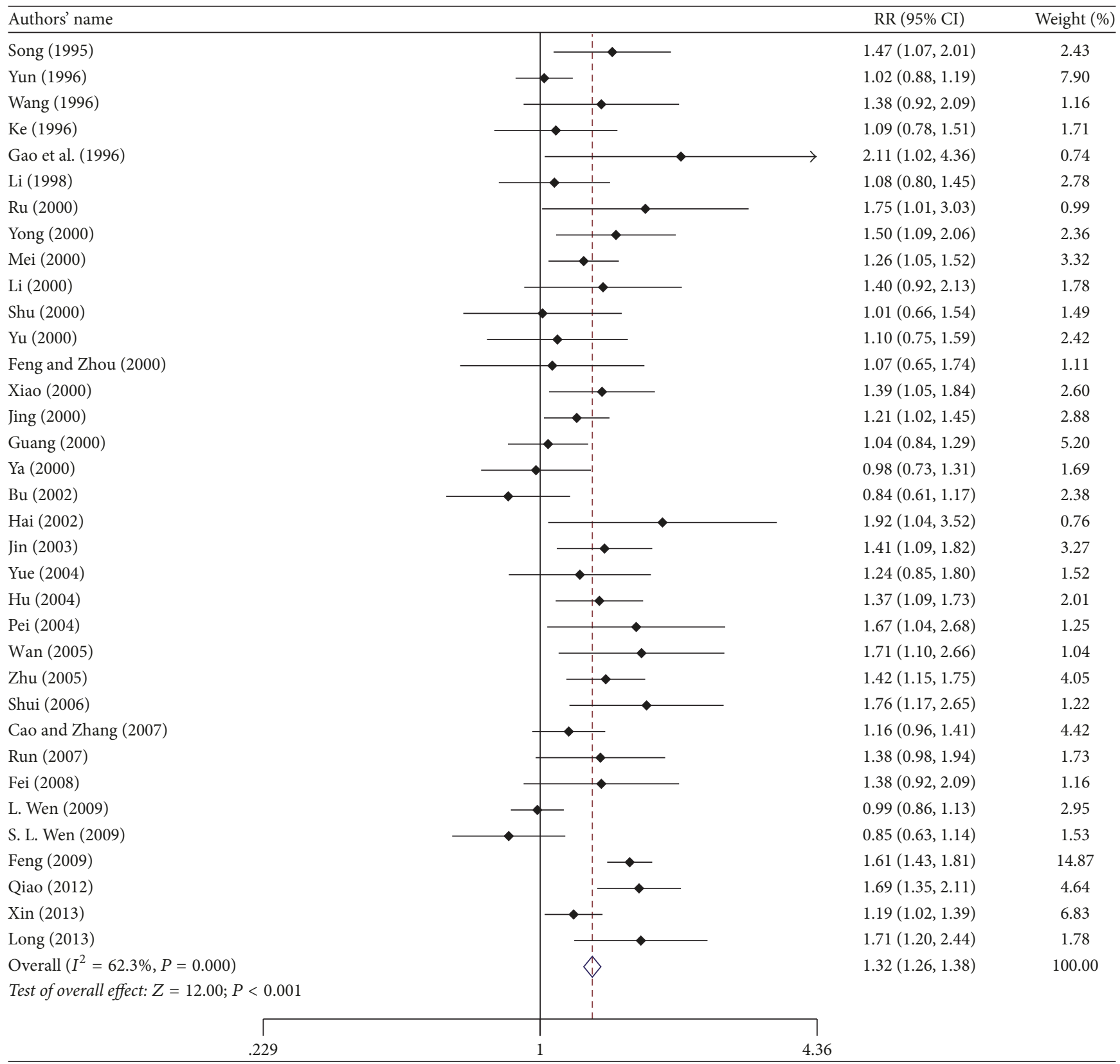

FIGURE 2: Relative risks for ECG improvement in the treatment and control groups.

in the treatment group compared with the control group (WMD $0.32 \mathrm{mmol} / \mathrm{L}, 95 \% \mathrm{CI} 0.07$ to $0.58 \mathrm{mmol} / \mathrm{L}$, and $P=$ $0.014)$, however with significant heterogeneity with respect to HDL levels $\left(I^{2}=87.5 \%\right)$ (Supplemental Figure S4). Sensitivity analysis did not reveal any single study as the source of heterogeneity. We did detect sample size $(P=0.013)$ as a contribution to the association between suxiao jiuxin pill and total triglyceride level, with no other significant factors being observed (Table 3 ).

3.5. Hemorheology. Six studies reported the levels of low-cut whole blood viscosity after treatment [39, 41, 43, 53, 57, 63]. There were significantly lower low-cut whole blood viscosity levels in the treatment group compared to the control group
(WMD $-1.57 \mathrm{mpa} \cdot \mathrm{s}, 95 \% \mathrm{CI}-2.50$ to $-0.65 \mathrm{mpa} \cdot \mathrm{s}$, and $P=$ $0.001)$. Significant heterogeneity was observed among these studies with respect to the level of low-cut whole blood viscosity $\left(I^{2}=86.8 \%\right)$ (Supplemental Figure S5). Six studies reported the levels of high-cut whole blood viscosity [39, 41, $43,53,57,63]$, which were significantly lower in the treatment group (WMD $-0.69 \mathrm{mpa} \cdot \mathrm{s}, 95 \% \mathrm{CI}-1.03$ to $-0.34 \mathrm{mpa} \cdot \mathrm{s}$, and $P<0.001)$. Significant heterogeneity was also observed among these studies with respect to the level of high-cut whole blood viscosity $\left(I^{2}=87.6 \%\right)$ (Supplemental Figure S6). Seven studies reported the levels of plasma viscosity $[33,39,41,43,54,57,63]$. There was no significant difference in WMD between the treatment and control groups (WMD $-0.03 \mathrm{mpa} \cdot \mathrm{s}, 95 \% \mathrm{CI}-0.07$ to $0.01 \mathrm{mpa} \cdot \mathrm{s}$, and $P=0.186)$ and 


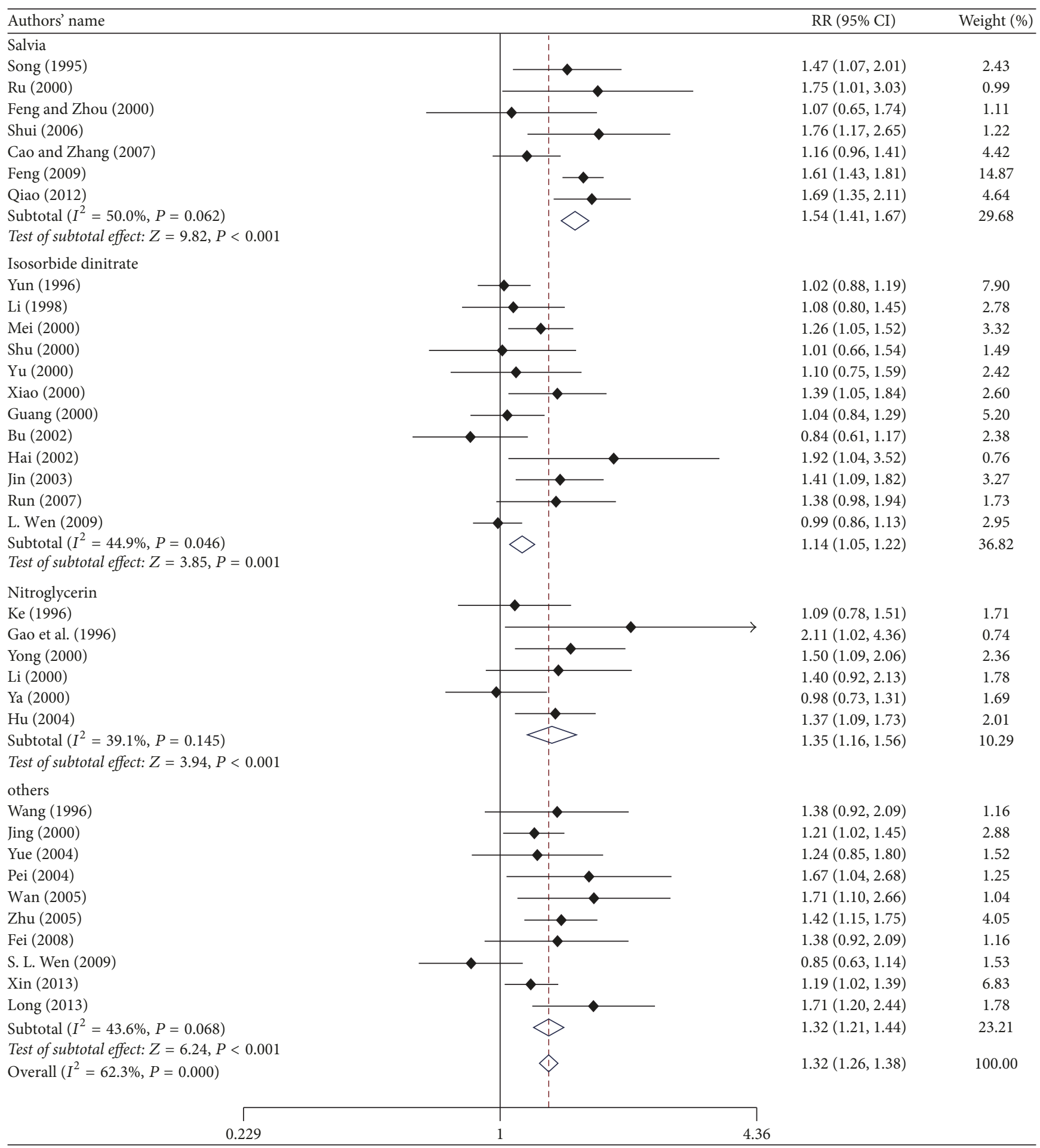

FIGURE 3: Relative risks for ECG improvement in the various subgroups.

no significant heterogeneity between studies among these studies with respect to the level of plasma viscosity $\left(I^{2}=4.9 \%\right)$ (Supplemental Figure S7). Four studies reported the levels of hematocrit $[33,54,57,63]$ and no significant differences were observed in WMD between the treatment and control groups (WMD $-1.24 \%, 95 \% \mathrm{CI}-3.26$ to $0.77 \%$, and $P=$ $0.227)$. There was significant heterogeneity among three studies with respect to the level of hematocrit $\left(I^{2}=84.3 \%\right)$ (Supplemental Figure S8). Four studies reported the levels of fibrinogen $[33,53,57,63]$ with no significant difference in WMD between the treatment and control groups (WMD $-0.76 \mathrm{~g} / \mathrm{L}, 95 \% \mathrm{CI}-1.32$ to $-0.20 \mathrm{~g} / \mathrm{L}$, and $P=0.008)$. There was significant heterogeneity among the three studies with respect to the fibrinogen level $\left(I^{2}=85 \%\right)$ (Supplemental 
TABLE 3: Metaregression based on sample size and mean age.

\begin{tabular}{lcc}
\hline Outcomes & Sample size & Mean age \\
\hline $\begin{array}{l}\text { Electrocardiogram } \\
\text { improvement }\end{array}$ & 0.206 & 0.059 \\
$\begin{array}{l}\text { Total cholesterol } \\
\text { Total triglycerides }\end{array}$ & 0.758 & 0.236 \\
$\begin{array}{l}\text { Total low-density } \\
\text { lipoprotein }\end{array}$ & 0.013 & 0.507 \\
Total high-density & 0.283 & 0.513 \\
lipoprotein & 0.715 & \\
Low-cutting whole blood & 0.917 & 0.789 \\
Viscosity & & 0.774 \\
High-cutting whole blood & 0.412 & \\
Viscosity & 0.075 & 0.621 \\
Plasma viscosity & 0.049 & 0.842 \\
Hematocrit & 0.890 & 0.345 \\
Fibrinogen & 0.554 & 0.772 \\
Adverse reactions & &
\end{tabular}

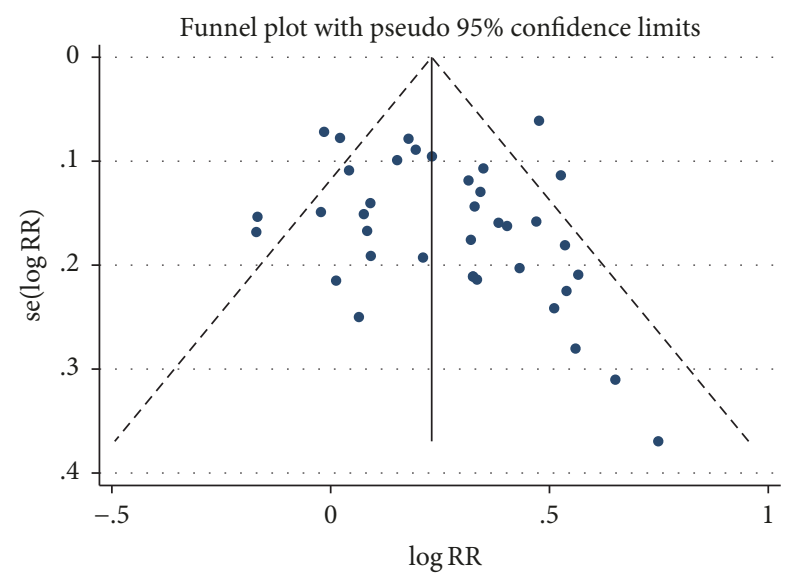

FIGURE 4: Funnel plot of studies included in the meta-analysis.

Figure S9). Sensitivity analysis was conducted by excluding each study individually and showed no substantial change in the overall trend. Furthermore, sample size and mean age were not correlated with treatment efficacy of suxiao jiuxin pills on hemorheology (Table 3).

3.6. Adverse Reactions. Fourteen studies reported the incidence of adverse reactions. The most common symptoms were mild headache, dizziness, and facial flushing. Most of these symptoms resolved spontaneously. There was no significant difference in the adverse reaction rates between the treatment and the control groups (RR 1.12, 95\% CI 0.50 to 2.51 , and $P=0.785$ ) and no significant heterogeneity with respect to adverse reaction rate $\left(I^{2}=49.1 \%\right)$ (Supplemental Figure S10). Additionally, meta-regression analyses suggested that both sample size and mean age were not associated with adverse reaction in the suxiao jiuxin pill treatment group (Table 3). The funnel plot was symmetrically distributed around the pooled effect size, which indicated the absence of significant publication bias in the included studies (Supplemental Figure S11). In addition, no publication bias was identified using the Egger test $(P=0.064)$ or Begg test $(P=$ $0.274)$.

\section{Discussion}

Based on our meta-analysis, we found that suxiao jiuxin pills could significantly improve ECG results in CHD patients compared with other therapies used in the selected studies. Suxiao jiuxin pills decreased the levels of total cholesterol and LDL, increased the levels of HDL, and lowered low-cut and high-cut whole blood viscosity. Other hemorheologyrelated parameters, such as plasma viscosity, hematocrit, and fibrinogen, showed the same tendency, but these changes were not statistically significant.

In recent years, several randomized clinical trials have been performed to evaluate the efficacy of suxiao jiuxin pill on CHD patients [17, 27, 28, 56]. Long studied 120 patients with unstable angina and found that suxiao jiuxin pills significantly improved both ECG results and symptoms compared with standard treatment [28]. Bu evaluated the benefits of suxiao jiuxin pills with isosorbide dinitrate on one hundred coronary heart disease (CAD) patients [44]. However, they did not find any significant differences between the groups with respect to ECG result improvement and angina relief. This may be due to the small sample size, different inclusion criteria, and differences in treatment strategy.

Two previous meta-analyses that explored the efficacy of suxiao jiuxin pills on $\mathrm{CHD}[65,66]$ found them to be effective in the treatment of angina pectoris, without any serious side effects. However, due to the limited sample sizes, low quality of the studies, and other potential confounding factors, the asymmetry funnel plot demonstrated the lack of reliability of these meta-analyses $[65,66]$. As a result, we conducted this updated meta-analysis of randomized controlled trials to further clarify the effects of suxiao jiuxin pills on $\mathrm{CHD}$ patients.

Suxiao jiuxin pills have been widely used in China for many years in patients with angina. It has two main effective components, borneol and Ligusticum chuanxiong Hort, which can be found mainly in the Sichuan province of China. Ligusticum chuanxiong Hort was first described in the 'Divine Husbandman's Materia Medica.' L. chuanxiong has long been regarded as a traditional Chinese medicine and has been added to food for its health benefits. The main chemical components of L. chuanxiong include essential oils, phenolic acids, and phthalide lactones $[67,68]$. Several researchers have demonstrated that L. chuanxiong could lower serum cholesterol and lipoprotein levels, reduce red blood cell deformability, and relieve angiotensin II-induced vascular smooth muscle cell proliferation. These unique roles may be due to the increase in nitric oxide and suppression of nuclear factor- $\kappa \mathrm{B}$ activation $[69,70]$. In addition, $L$. chuanxiong has a direct vasodilatory effect on isolated aortic rings in rats [71]. The mechanisms of this effect are related to the opening of SK (Ca) and $\mathrm{ATP}_{\mathrm{K}}$ channels, the reduction of ET-1, and the formation of reactive oxygen species (ROS) [72, 73]. Recent evidence has suggested that $L$. chuanxiong may exert 
antiplatelet effects by inhibiting the vWF-mediated process of platelet thrombus formation.

Borneol is a fragrant ingredient used in decorative cosmetics and is widely regarded as an adjuvant in Chinese herbs [74]. Several animal studies have demonstrated that borneol can dilate coronary arteries and improve coronary circulation. In addition, studies have demonstrated that borneol can inhibit the inflammatory response in animal models [75]. As a result, borneol is widely used in China for the treatment of CHD patients in clinical practice.

We found that the main side effects of suxiao jiuxin pills were mild headaches, dizziness, and facial flushing, all of which resolved spontaneously. The adverse reaction incidence associated with suxiao jiuxin pill was not significantly different from those associated with other treatments. Evidence suggests that suxiao jiuxin pills are safe with no adverse effects.

As in many meta-analyses, there were several limitations to our study. Most of the clinical studies included in our metaanalysis were of poor quality based on their Jadad scores. Only a few studies reported detailed research methodology, a factor that could decrease the reliability of this meta-analysis. In addition, all of the included studies were from Chinese publications, and this may be a source of bias. Significant heterogeneity between studies was noted in our meta-analysis even after subgroup analysis. Different study quality, sample size, usage of suxiao jiuxin pill, and control groups may contribute to this heterogeneity. In this study, we did not analyze cardiovascular death or drug-related complications.

In summary, our meta-analysis demonstrated that suxiao jiuxin pills improved ECG results and lipid profiles better compared with nitroglycerin, isosorbide dinitrate, salvia, and other Chinese herbal pills. They also decreased low-cut and high-cut whole blood viscosity.

\section{Conclusions}

Suxiao jiuxin pills can effectively decrease the lipid profiles and improve hemorheology parameters in CHD patients. This is due to the effects of their components (borneol and $L$. chuanxiong), which may improve coronary artery circulation and ECG results.

\section{Conflicts of Interest}

The authors declare no conflicts of interest regarding the publication of this article.

\section{Supplementary Materials}

Figure S1: mean difference in total cholesterol in the treatment and control groups. Figure S2: mean difference in total triglycerides in the treatment and control groups. Figure S3: mean difference in total low-density lipoprotein in the treatment and control groups. Figure S4: mean difference in total high-density lipoprotein in the treatment and control groups. Figure S5: mean difference in low-cut whole blood viscosity in the treatment and control groups. Figure S6: mean difference in high-cut whole blood viscosity in the treatment and control groups. Figure S7: mean difference in plasma viscosity in the treatment and control groups. Figure S8: mean difference in hematocrit in the treatment and control groups. Figure S9: mean difference in the fibrinogen in the treatment and control groups. Figure S10: relative risks for adverse reactions in the treatment and control groups. Figure S11: funnel plot of studies with reported adverse reactions included in the meta-analysis. (Supplementary Materials)

\section{References}

[1] S. S. Yoon, C. F. Dillon, K. Illoh, and M. Carroll, "Trends in the Prevalence of Coronary Heart Disease in the U.S.: National Health and Nutrition Examination Survey, 2001-2012," American Journal of Preventive Medicine, vol. 51, no. 4, pp. 437445, 2016.

[2] R. K. Zeidan, R. Farah, M. N. Chahine et al., "Prevalence and correlates of coronary heart disease: First population-based study in Lebanon," Vascular Health and Risk Management, vol. 12, pp. 75-84, 2016.

[3] M. Haim, M. Hoshen, O. Reges, Y. Rabi, R. Balicer, and M. Leibowitz, "Prospective national study of the prevalence, incidence, management and outcome of a large contemporary cohort of patients with incident non-valvular atrial fibrillation," Journal of the American Heart Association, vol. 4, no. 1, article e001486, 2015.

[4] W. B. Kannel, R. D. Abbott, D. D. Savage, and P. M. McNamara, "Coronary heart disease and atrial fibrillation: The Framingham Study," American Heart Journal, vol. 106, no. 2, pp. 389-396, 1983.

[5] S. Lokshyn, C. Mewis, and V. Kuhlkamp, "Atrial fibrillation in coronary artery disease," International Journal of Cardiology, vol. 72, no. 2, pp. 133-136, 2000.

[6] J. E. Otterstad, B.-A. Kirwan, J. Lubsen et al., "Incidence and outcome of atrial fibrillation in stable symptomatic coronary disease," Scandinavian Cardiovascular Journal, vol. 40, no. 3, pp. 152-159, 2006.

[7] N. J. Patel, A. Patel, K. Agnihotri et al., "Prognostic impact of atrial fibrillation on clinical outcomes of acute coronary syndromes, heart failure and chronic kidney disease," World Journal of Cardiology, vol. 7, no. 7, pp. 397-403, 2015.

[8] P. Zhang, R. Qiu, R. Shen, and J. Liang, "Clinical observation in 40 cases of postmenopausal coronary heart disease treated with Yanghuo Sanzi Tang," Journal of Traditional Chinese Medicine, vol. 23, no. 3, pp. 182-184, 2003.

[9] Z. Lu, Y. Zhang, P. Zhuang et al., "Protective effect of Suxiao jiuxin pill, a traditional Chinese medicine, against acute myocardial ischemia in dogs," BMC Complementary and Alternative Medicine, vol. 15, no. 1, article no. 373, 2015.

[10] J. Zhang, P. Zhuang, Z. Lu et al., "Suxiaojiuxin pill enhances atherosclerotic plaque stability by modulating the MMPs/TIMPs balance in ApoE-deficient Mice," Journal of Cardiovascular Pharmacology, vol. 64, no. 2, pp. 120-126, 2014.

[11] X.-Y. Bai, P. Zhang, Q. Yang et al., "Suxiao Jiuxin Pill induces potent relaxation and inhibition on contraction in human artery and the mechanism," Evidence-Based Complementary and Alternative Medicine, vol. 2014, Article ID 956924, 11 pages, 2014.

[12] C.-S. Li, Z.-Q. Qu, S.-S. Wang et al., "Effects of suxiao Jiuxin Pill on oxidative stress and inflammatory response in rats with 
experimental atherosclerosis," Journal of Traditional Chinese Medicine, vol. 31, no. 2, pp. 107-111, 2011.

[13] S. F. Dong and Z. G. Zhu, "Determination of the contents of $\mathrm{Ca}, \mathrm{Mg}, \mathrm{Fe}, \mathrm{Cu}$ and $\mathrm{Zn}$ in suxiao jiuxin pill and the analysis of $\mathrm{Ca} / \mathrm{Mg}$ and $\mathrm{Cu} / \mathrm{Zn}$ values," Guang Pu Xue Yu Guang Pu Fen Xi, vol. 22, no. 3, pp. 478-479, 2002.

[14] Q. Guo, J. Zhang, Y. Li, and G. Zhang, "Study on antiatherosclerotic effect of suxiao jiuxin pill and its mechanism," African Journal of Traditional, Complementary and Alternative Medicines, vol. 11, no. 1, pp. 97-102, 2014.

[15] X.-L. Wang, Y.-M. Liu, and G.-J. Zhu, "Effects of suxiao jiuxin pill on patients with acute coronary syndrome undergoing early percutaneous coronary intervention," Zhongguo Zhong Xi Yi Jie He Za Zhi, vol. 32, no. 11, pp. 1483-1487, 2012.

[16] S. H. Cao and J. Y. Zhang, "Effects of suxiao jiuxin pill on 105 coronary heart diseases," Chinese Medicine, vol. 29, no. 4, pp. 486-488, 2007.

[17] K. X. Qiao, "Effect of suxiao jiuxin pill combined with Trimetazidine on patients with Angina," Journal of North Pharmacy, vol. 10, article 38, 2012.

[18] F. C. Pei, "Effects of continuous suxiao jiuxin pill on 60 patients with coronary heart diseases," Chinese Journal of Geriatrics, vol. 2, no. 3, pp. 24-26, 2004.

[19] X. Duan, L. Zhou, T. Wu et al., "Chinese herbal medicine suxiao jiuxin wan for angina pectoris." Cochrane Database of Systematic Reviews (Online), no. 1, p. CD004473, 2008.

[20] D. Moher, A. Liberati, J. Tetzlaff, D. G. Altman, and P. Group, "Preferred reporting items for systematic reviews and metaanalyses: the PRISMA Statement," Open Medicine, vol. 3, no. 3, pp. e123-e130, 2009.

[21] S. C. Smith Jr., E. J. Benjamin, R. O. Bonow et al., "AHA/ACCF secondary prevention and risk reduction therapy for patients with coronary and other atherosclerotic vascular disease: 2011 update: a guideline from the American Heart Association and American College of Cardiology Foundation endorsed by the World Heart Federation and the Preventive Cardiovascular Nurses Association," Journal of the American College of Cardiology, vol. 58, no. 23, pp. 2432-2446, 2011.

[22] S. C. Smith Jr., E. J. Benjamin, R. O. Bonow et al., "AHA/ACCF Secondary Prevention and Risk Reduction Therapy for Patients with Coronary and other Atherosclerotic Vascular Disease: American College of Cardiology Foundation," Circulation, vol. 124, no. 22, pp. 2458-2473, 2011.

[23] A. R. Jadad, R. A. Moore, D. Carroll et al., "Assessing the quality of reports of randomized clinical trials: Is blinding necessary?" Controlled Clinical Trials, vol. 17, no. 1, pp. 1-12, 1996.

[24] S. G. Thompson and J. P. T. Higgins, "How should metaregression analyses be undertaken and interpreted?" Statistics in Medicine, vol. 21, no. 11, pp. 1559-1574, 2002.

[25] J. J. Deeks, J. P. T. Higgins, and D. G. Altman, "Analyzing data and undertaking meta-analyses," in Cochrane Handbook for Systematic Reviews of Interventions 5.0.1, J. Higgins and S. Green, Eds., The Cochrane Collaboration: Chap 9, Oxford, UK, 2008.

[26] J. P. T. Higgins, S. G. Thompson, J. J. Deeks, and D. G. Altman, "Measuring inconsistency in meta-analyses," British Medical Journal, vol. 327, no. 7414, pp. 557-560, 2003.

[27] H. Xin, "Effect of suxiao jiuxin pill on unstable angina," Chinses Community Docotors, vol. 41, pp. 47-48, 2013.

[28] J. Q. Long, "Effect of suxiao jiuxin pill on unstable angina," Chinses Community Docotors, vol. 7, article 23, 2013.
[29] Y. C. Gao, A. X. Liu, D. F. Yin, C. H. Chen, and R. X. Gao, "Effect of suxiao jiuxin pill on 105 patients with angina," Journal of Emergency in Traditional Chinese Medicine, vol. 2, pp. 74-75, 1996.

[30] J. H. Yong, "Effect of suxiao jiuxin pill on 84 patients with angina," Tianjin Pharmacy, vol. 12, no. B11, pp. 42-43, 2000.

[31] G. Hu, "Effect of suxiao jiuxin pill on patients with coronary heart disease," Clinical Journal of Traditional Chinese Medicine, vol. 16, no. 1, pp. 53-54, 2004.

[32] F. J. Ke, "Effect of suxiao jiuxin pill on 36 patients with angina," Journal of Emergency in Traditional Chinese Medicine, vol. 5, no. 3, article 118, 1996.

[33] Y. W. Pei, "Comparison between suxiao jiuxin pill and Isosorbide Mononitrate on angina," Modern Medicine and Health, vol. 19, no. 3, article 274, 2003.

[34] X. Z. Ya, "Effect of suxiao jiuxin pill on eldly patients with angina," Tianjin Pharmacy, vol. 12, no. B11, pp. 46-47, 2000.

[35] J. Z. Li, "Effect of suxiao jiuxin pill on patients with angina," Tianjin Pharmacy, vol. 12, no. B11, article 62, 2000.

[36] A. Li, "Effect of suxiao jiuxin pill on 103 patients with angina," Qingdao Medical Journal, vol. 6, pp. 39-40, 1998.

[37] G. Jin, "Effect of suxiao jiuxin pill on 98 patients with angina," Journal of Chinese General Practice, vol. 6, no. 3, pp. 250-251, 2003.

[38] Y. G. Yun, "Effect of suxiao jiuxin pill on patients with angina," Journal of Emergency in Traditional Chinese Medicine, vol. 5, no. 3, pp. 115-116, 1996.

[39] H. Mei, "Effect of suxiao jiuxin pill on 68 patients with angina," Tianjin Pharmacy, vol. 12, no. B11, pp. 36-37, 2000.

[40] P. L. Yu, "Effect of suxiao jiuxin pill on patients with angina," Tianjin Pharmacy, vol. 12, no. B11, article 47, 2000.

[41] C. L. Xiao, "Effect of suxiao jiuxin pill on 78 patients with angina," Tianjin Pharmacy, vol. 12, no. B11, pp. 811-812, 2000.

[42] L. Wen, "Effect of suxiao jiuxin pill combined with compound danshen dripping pill on patients with angina," Shanxi Journal of Traditional Chinese Medicine, vol. 25, no. 8, pp. 12-13, 2009.

[43] S. Hai, "Effect of suxiao jiuxin pill on 40 patients with angina," Chinese Medincie, vol. 24, no. 11, pp. 852-854, 2002.

[44] C. S. Bu, "Comparison between suxiao jiuxin pill and Isosorbide dinitrate on angina," Chinese Journal of Natural Medicine, vol. 4, no. 3, pp. 185-186, 2002.

[45] Y. T. Guang, "Effect of suxiao jiuxin pill on 124 patients with angina," Tianjin Pharmacy, vol. 12, no. 1, pp. 40-41, 2000.

[46] L. T. Run, "Comparison between suxiao jiuxin pill and Isosorbide dinitrate on angina," Journal of Snake, vol. 19, no. 1, article 37, 2007.

[47] D. Y. Shu, "Effect of suxiao jiuxin pill on patients with unstable angina," Tianjin Pharmacy, vol. 12, no. B11, pp. 28-29, 2000.

[48] L. Feng and Y. P. Zhou, "Effect of suxiao jiuxin pill on patients with angina," Journal of Emergency in Traditional Chinese Medicine, vol. 9, no. 1, pp. 4-6, 2000.

[49] H. S. Feng, "Effect of suxiao jiuxin pill on patients with angina," Beijing Journal of Traditional Chinese Medicine, vol. 28, no. 9, pp. 25-26, 2009.

[50] X. W. Shui, "Effect of suxiao jiuxin pill and compound danshen dripping pill on patients with angina," Jiangxi Medicine, vol. S1, pp. 1073-1074, 2006.

[51] Z. J. Song, "Effect of suxiao jiuxin pill on patients with angina," Chinese Journal of Integrated Traditional and Western Medicine in Intensive and Critical Care, vol. 2, pp. 83-84, 1995. 
[52] G. H. He, "Effect of suxiao jiuxin pill on patients with angina," Chinese Journal of Integrated Traditional and Western Medicine in Intensive and Critical Care, vol. 5, pp. 214-215, 1995.

[53] X. Z. Ma, "Effect of suxiao jiuxin pill on 83 patients with angina," Modern Medicine and Health, vol. 20, no. 21, pp. 2211-2212, 2004.

[54] W. Q. Guo, "Effect of suxiao jiuxin pill on patients with coronary heart disease," Chinese Community Doctors, vol. 14, article 11, 2012.

[55] B. J. Ru, "Effect of suxiao jiuxin pill on patients with angina," Tianjin Pharmacy, vol. 12, no. B11, article 46, 2000.

[56] X. J. Li, "Clinical observation on effect of suxiao jiuxin pill in treating angina pectoris of coronary heart disease," Strait Pharmacy, vol. 2, pp. 98-99, 2015.

[57] K. J. Duan and X. Y. Yang, "Effect of suxiao jiuxin pill on 40 patients with angina," Tianjin Journal of Traditional Chinese Medicine, vol. 19, no. 1, pp. 20-21, 2002.

[58] S. L. Wen, "Comparison between suxiao jiuxin pill and Tongxinluo pill on angina," Chinese Community Docotor, vol. 6, article 62, 2009.

[59] W. Wan, "Comparison between suxiao jiuxin pill and Guanxinsuhe pill on angina," Medicine Industry Information, vol. 2, no. 17, article 59, 2005.

[60] D. P. Wang, "Comparison between suxiao jiuxin pill and shexiang baoxin pill on angina," Shanghai Medicine, vol. 12, pp. 25-26, 1996.

[61] W. Fei, "Comparison between suxiao jiuxin pill and shexiang baoxin pill on angina," Medicine Review, vol. 14, no. 19, pp. 30223024, 2008.

[62] S. Z. Yue, "Effect of suxiao jiuxin pill on 78 patients with angina and heart failure," Journal of Practical Medical Techniques, vol. 6, pp. 31-32, 2004.

[63] D. Y. Zhu, "Effect of suxiao jiuxin pill on 118 patients with angina," Henan Chinese Medicine, vol. 25, no. 9, pp. 76-77, 2005.

[64] X. Y. Jing, "Effect of suxiao jiuxin pill on 102 patients with coronary heart disease," Tianjin Pharmacy, vol. 12, no. B11, pp. 37-38, 2000.

[65] W. W. Wei, "Efficacy of Suxiaojiuxinwan vs. Isosorbide Dinitratein patients with angina pectoris: a meta-analysis," Chinese Journal of Evidence-Based Cardiovascular Medicine, vol. 3, pp. 298-303, 2015.

[66] F. R. Xiao, "Meta-analysis of Suxiao Jiuxin Pill on Acute Coronary Syndrome," Journal of Emergency in Traditional Chinese Medicine, vol. 3, pp. 380-383+425, 2016.

[67] W. Li, Y. Tang, Y. Chen, and J.-A. Duan, "Advances in the chemical analysis and biological activities of chuanxiong," Molecules, vol. 17, no. 9, pp. 10614-10651, 2012.

[68] X. Ran, L. Ma, C. Peng, H. Zhang, and L.-P. Qin, "Ligusticum chuanxiong Hort: a review of chemistry and pharmacology," Pharmaceutical Biology, vol. 49, no. 11, pp. 1180-1189, 2011.

[69] D. C. Ren, N. Y. Yang, S. H. Qian, N. Xie, X. M. Zhou, and J. A. Duan, "Chemical study on aerial parts of Ligusticum chuanxiong," Zhongguo Zhong Yao Za Zhi, vol. 32, no. 14, pp. 1418-1420, 2007.

[70] X.-Y. Ren, Q.-R. Ruan, D.-H. Zhu, M. Zhu, Z.-L. Qu, and J. Lu, "Tetramethylpyrazine inhibits agiontensin II-induced nuclear factor-kappaB activation and bone morphogenetic protein-2 downregulation in rat vascular smooth muscle cells," Sheng $\mathrm{Li}$ Xue Bao, vol. 59, no. 3, pp. 339-344, 2007.

[71] C.-C. Tsai, T.-Y. Lai, W.-C. Huang, I.-M. Liu, and J.-T. Cheng, "Inhibitory effects of potassium channel blockers on tetramethylpyrazine-induced relaxation of rat aortic strip in vitro," Life Sciences, vol. 71, no. 11, article no. 8922, pp. 1321-1330, 2002.

[72] Y. Z. Hou, G. R. Zhao, J. Yang, Y. J. Yuan, G. G. Zhu, and R. Hiltunen, "Protective effect of Ligusticum chuanxiong and Angelica sinensis on endothelial cell damage induced by hydrogen peroxide," Life Sciences, vol. 75, no. 14, pp. 1775-1786, 2004.

[73] K.-L. Wong, K.-C. Wu, R. S.-C. Wu, Y.-H. Chou, T.-H. Cheng, and H.-J. Hong, "Tetramethylpyrazine inhibits angiotensin IIincreased $\mathrm{NAD}(\mathrm{P}) \mathrm{H}$ oxidase activity and subsequent proliferation in rat aortic smooth muscle cells," American Journal of Chinese Medicine, vol. 35, no. 6, pp. 1021-1035, 2007.

[74] E. W. E. C. Chan and S. K. U. Wong, "Phytochemistry and pharmacology of ornamental gingers, Hedychium coronarium and Alpinia purpurata: a review," Journal of Integrative Medicine, vol. 13, no. 6, pp. 368-379, 2015.

[75] S. P. Bhatia, C. S. Letizia, and A. M. Api, "Fragrance material review on borneol," Food and Chemical Toxicology, vol. 46, no. 11, pp. S77-S80, 2008. 


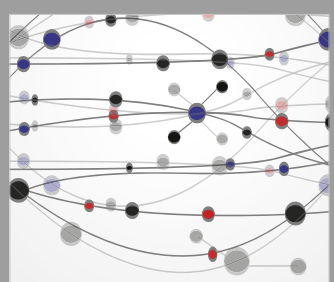

The Scientific World Journal
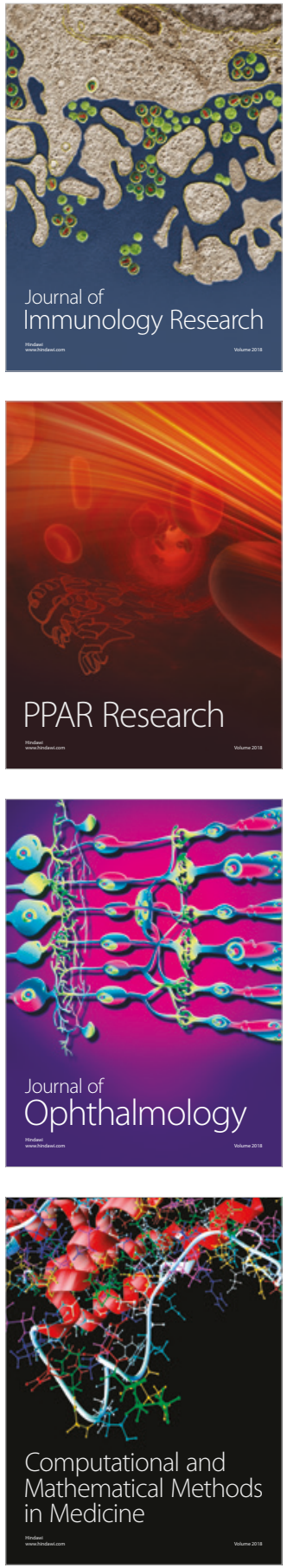

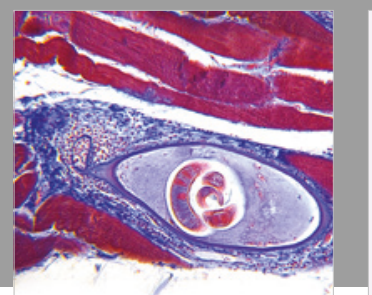

Gastroenterology Research and Practice

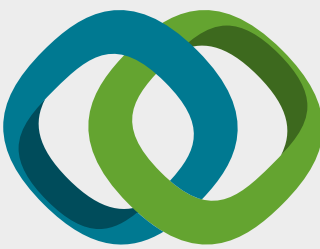

\section{Hindawi}

Submit your manuscripts at

www.hindawi.com
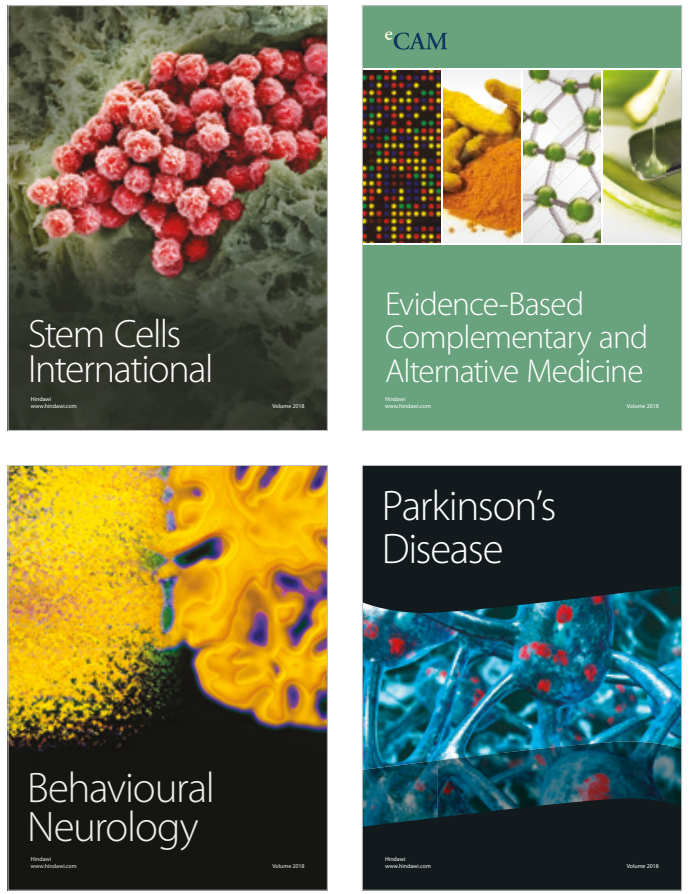

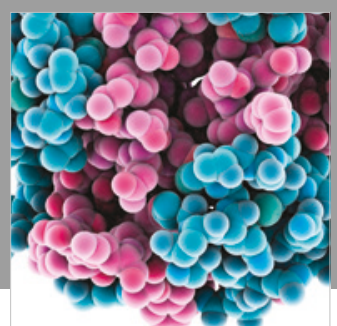

ournal of

Diabetes Research

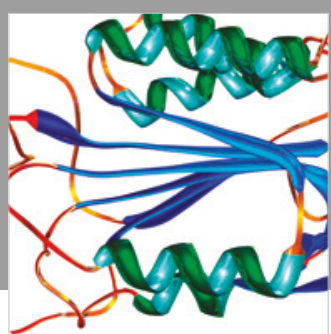

Disease Markers
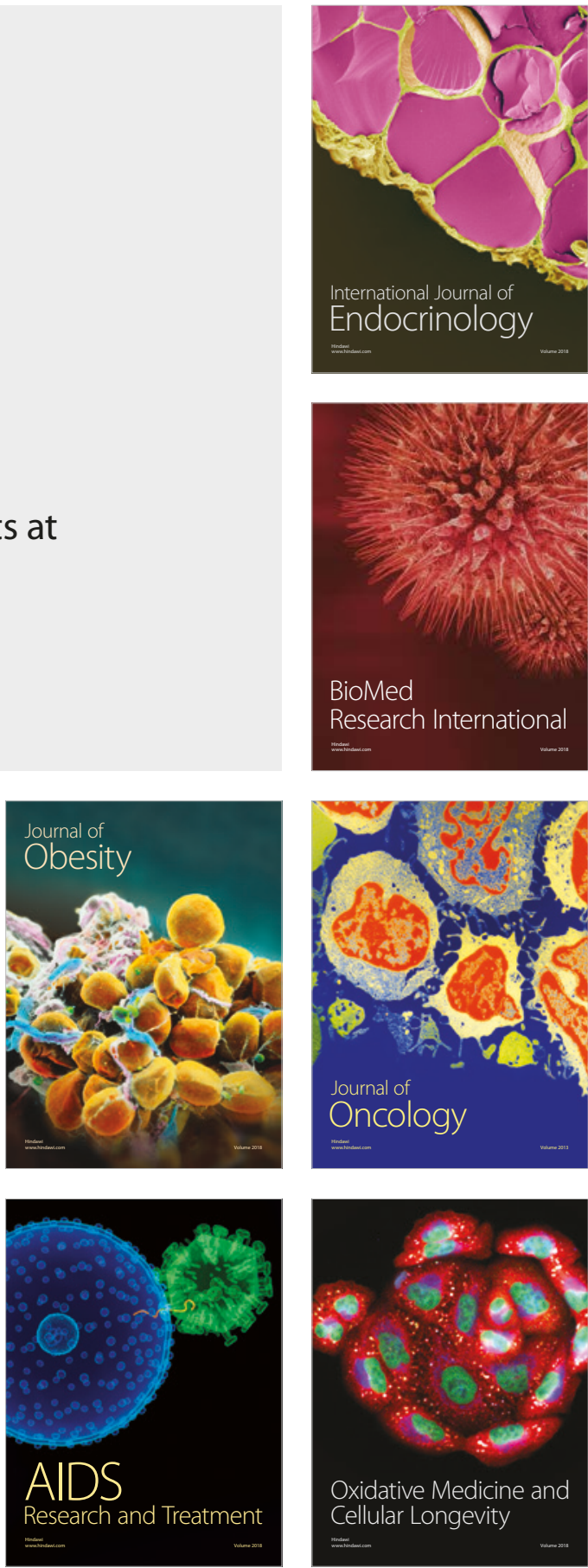